\title{
High-resolution ultrasound imaging using unified pixel-based and filtered delay multiply and sum beamforming
}

\author{
Hao Guo \\ The School of Biological Science \\ and Medical Engineering \\ Southeast University \\ Nanjing, China \\ haogseu@seu.edu.cn \\ Nghia Q. Nguyen \\ Department of Engineering, \\ University of Cambridge \\ Cambridge University - Nanjing Centre \\ of Technology and Innovation \\ Cambridge, United Kingdom \\ nqn20@cam.ac.uk
}

\author{
Hui-Wen Xie \\ The School of Biological Science \\ and Medical Engineering \\ Southeast University \\ Nanjing, China \\ huiwen_xie@seu.edu.cn \\ Richard W. Prager \\ Department of Engineering, \\ University of Cambridge \\ Cambridge University - Nanjing Centre \\ of Technology and Innovation \\ Cambridge, United Kingdom \\ rwp@eng.cam.ac.uk
}

\author{
Guang-Quan Zhou \\ The School of Biological Science \\ and Medical Engineering \\ Southeast University \\ Nanjing, China \\ guangquan.zhou@seu.edu.cn
}

\begin{abstract}
Unified pixel-based (PB) beamforming has previously been implemented for ultrasound imaging. It was shown to offer significant enhancements in lateral resolution compared to conventional dynamic focusing. Yet, there is still interference from clutter and high sidelobe artefacts that limit the the contrast resolution. In this paper, we propose a combination of the unified pixel-based approach with filtered delay multiply and sum (FDMAS) as a way of further improving the image quality. This hybrid strategy leverages the spatial coherence among signals collected from regions far from the focal depth. As a result, it helps suppress artefacts from the sidelobes and clutter, which can enhance the contrast resolution on the generated image. The new beamformer is evaluated on both simulation and data from an experimental study. The results show that the proposed approach can significantly improve the lateral resolution and image contrast over the unified PB beamformer. Compared to the F-DMAS, it maintains the high contrast resolution while generating uniform brightness over the entire imaging region.

Index Terms - ultrasound, beamforming, filtered delay multiply and sum, pixel-based, image quality.
\end{abstract}

\section{INTRODUCTION}

The goal of any ultrasound beamformer is to create a narrow and uniform pulse over the entire imaging region [1]. In most conventional ultrasound systems, however, the image is still generated with a fixed-focus beam on transmit and dynamic focusing on receive. As a result, the generated image has the optimal resolution only around the focal depth. Attempts to enhance the image quality usually lead to the accumulation of data acquired in multiple transmits. In this approach, the signals to be superimposed are extracted from received waveforms by using the time-delay calculation. The calculation is based on an assumption that the transmit waveshape at each imaging point is a spherical pulse propagating from a single element or the center of an active subaperture $[2,3]$.

In a previous study [4], we developed a new unified pixelbased (PB) beamformer by modeling the transmit waveshape as a superposition of two spherical pulses, corresponding to the minimal and maximal distances from the imaging point to the active subaperture. This model is developed through our field pattern analysis. Based on the model, we have formed a new time-delay calculation that extracts the best possible data associated with each of these pulses. The imaging results show significant improvements in lateral resolution and speckle patterns. There are, however, still clutter and noticeable sidelobe artefacts in the near- and far-field of the imaging regions that compromise the image contrast. Finding a new method to suppress these artefacts and further enhance the image quality is the main purpose of this work.

In [5], Matrone et al. introduced a novel nonlinear beamformer, called filtered delay multiply and sum (F-DMAS), that improves the contrast resolution of the generated image. In this approach, the echo signals, extracted from received radiofrequency (RF) data, are multiplied by each other before taking a square root and calculating the summation. The method exploits the spatial coherence among all the received signals to enhance the contrast resolution. However, there is a strong brightness around the focal depth which may indicate the loss of coherence among selected signals in the other regions of the generated images.

In this study, we explore a hybrid algorithm between the FDMAS and unified PB to further improve image quality. The combination can increase the spatial coherence information 


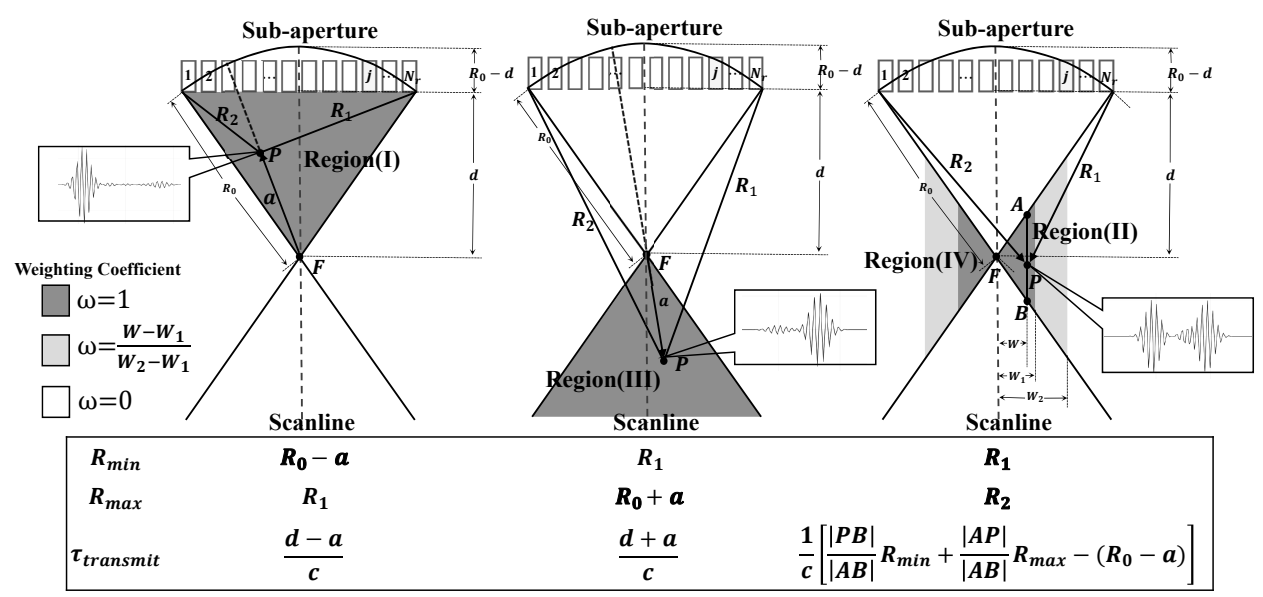

Fig. 1. Geometries for the transmit time-delay calculations at point $\mathrm{P}$ with a focused beam. Transmit waveshape at $\mathrm{P}$ in different regions is shown in the rectangular box.

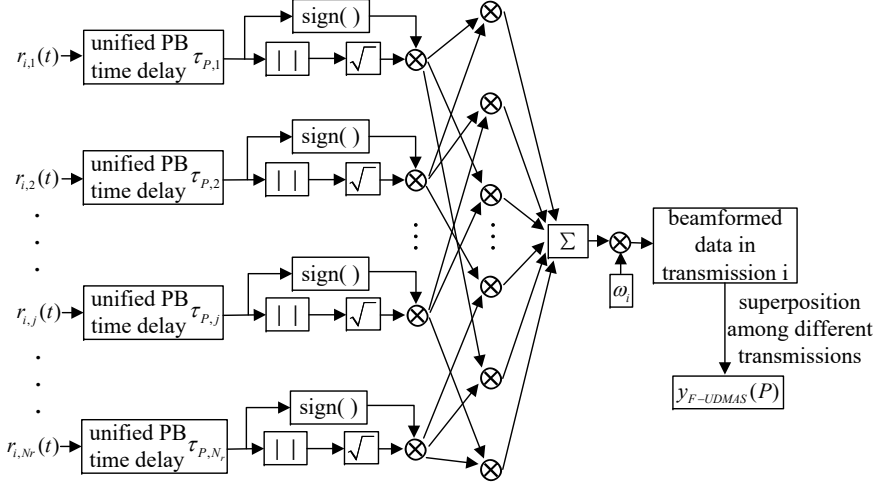

Fig. 2. The F-UDMAS beamformer block diagram. The specific flow of this algorithm in one transmission is shown in the left half of this diagram.

through better alignments of signals extracted at imaging points in the near- and far-field regions. As a result, it should correct the loss of brightness on the F-DMAS image when moving away from the focal depth, as reported in [6]. We name the combination unified pixel-based and filtered delay multiply and sum (F-UDMAS) beamforming.

\section{MethodS}

\section{A. Unified Pixel-Based Beamforming}

We begin by summarizing the unified pixel-based beamformer developed in [4]. It involves data generation similar to that of synthetic aperture (SA) imaging, given by

$$
y_{\text {unified-PB }}=\sum_{i=1}^{N_{t}} \omega_{i} \sum_{j=1}^{N_{r}} r_{i, j}\left(\tau_{P}(i, j)\right) \text {, }
$$

where $\mathrm{P}$ is the imaging point, $N_{t}$ is the number of transmits, $N_{r}$ is the number of receive elements, $r_{i, j}$ is the RF data received by the $j$ th elements in the $i$ th transmission, $\omega_{i}$ is the weighting coefficient and $\tau_{P}$ is the time-delay at $\mathrm{P}$.

A major difference of the unified PB from SA imaging is the strategy to calculate time-delay $\tau_{P}$. The transmit time-delay is varied depending on the position of $\mathrm{P}$ with respect to the subaperture as illustrated in Fig. 1, while the receive timedelay can be calculated directly from the distance between imaging point $\mathrm{P}$ and each of the received elements.
The field of view can be divided into four regions (I) to (IV), defined clockwise around the focal point $\mathrm{F}$. In all four regions, the transmit waveshape can be approximately as a superposition of two spherical pulses, that correspond to the minimal and maximal distances $\left(R_{\min }\right.$ and $\left.R_{\max }\right)$ from the imaging point to the transmit aperture. In regions (I) and (III), the waveshape is dominated by the first and second pulses, respectively. Thus we assign $\tau_{P}$ to $\tau_{P, \min }$ in region (I) and to $\tau_{P, \max }$ in region (III). These are the time-delays corresponding to those pulses. In regions (II) and (IV), the two pulses have similar magnitude. We set $\tau_{P}$ as a linear weighted sum of $\tau_{P, \min }$ and $\tau_{P, \max }$. It helps to have a smooth transition from region (I) to region (III) when moving across the focal depth.

This new time-delay calculation is shown to improve the lateral resolution significantly. Details of the analysis and imaging results are provided in [4].

\section{B. Filtered Delay Multiply and Sum Beamforming}

The F-DMAS algorithm is developed from conventional dynamic focusing. The conventional method dynamically aligns the echo signals $r_{j}(t)$ at individual imaging points with timedelay $t$, and then sums them together. Thus, the output signal is given by

$$
y=\sum_{j=1}^{N_{r}} r_{j}(t)
$$

The F-DMAS beamformer is a nonlinear spatial-coherence method that helps achieve high-quality ultrasound images. It extracts the signals from individual receive channels in the same way that conventional dynamic focusing does. The main difference in F-DMAS is the multiplying and summation of the signed and squared signals $\hat{r}_{m}(t)$ and $\hat{r}_{n}(t)$ in pairs. Thus, the beamformer output is given by

$$
y_{F-D M A S}=\sum_{m=1}^{N_{r}-1} \sum_{n=m+1}^{N_{r}} \hat{r}_{m}(t) \hat{r}_{n}(t),
$$

where $\hat{r}_{m}(t)=\operatorname{sign}\left(r_{m}(t)\right) \cdot \sqrt{\left|r_{m}(t)\right|}$, and $m$ and $n$ are the indexes of two individual receiving elements. Because of the multiplication, the output signal is modulated to a frequency band around $2 f_{0}$ where $f_{0}$ is the center frequency of the transmit beam. Thus, the signal needs to go through a 
bandpass filter centered at $2 f_{0}$ before the envelope detection. The diagram of this beamformer is depicted in Fig. 2 [5].

\section{Unified pixel-based and filtered delay multiply and sum Beamforming}

In $[5,7]$, it is reported that the F-DMAS mainly has impact on the contrast resolution and has an uneven brightness distribution as a function of imaging depth. Based on our analysis, we conjecture that the conventional time-delay calculation may limit the spatial resolution of the F-DMAS beamformer. As a result, it should be possible to improve the quality of the generated image by adopting the new time-delay calculation that we develop for unified PB beamforming.

Thus, we combine the time-delay calculation, depicted in Fig. 1, with the signal generation, depicted in Fig. 2 for data generated within one transmit. The results are accumulated across multiple transmits to generate the F-UDMAS beamformed signals. The entire process within one transmit is summarized by

$$
y_{F-U D M A S}=\sum_{m=1}^{N_{r}-1} \omega_{m} \sum_{n=m+1}^{N_{r}} \hat{r}_{m}\left(\tau_{P}(m)\right) \hat{r}_{n}\left(\tau_{P}(n)\right),
$$

Similar to the F-DMAS beamformer, the F-UDMAS beamformed signals also need to be filtered with a bandpass filter centered at $2 f_{0}$.

\section{RESULTS}

\section{A. Data Acquisition and Performance Metrics}

We demonstrate the new F-UDMAS on both simulated and experimental data. The experimental data is obtained from the Verasonics Vantage-256 system (Verasonics, Kirkland, WA, USA) that uses the L7-4 probe (128 elements, Philips) to scan a tissue-mimicking phantom. The simulation is generated with Field-II program [8] and also based on the parameters of the Verasonics Vantage-256 system and L7-4 probe. We use an excitation signal with a center frequency $f_{0}$ of $5.2083 \mathrm{MHz}$ and $67 \%$ bandwidth. A subaperture of 64 elements are set active on both transmit and receive. Data is acquired with a sampling frequency of $41.66 \mathrm{MHz}$, equivalent to $8 f_{0}$. The transmit beam is shifted by an element pitch of $0.298 \mathrm{~mm}$ laterally after each pulse-echo sequence. During the scan conversion, the envelope data is upsampled with a five-fold finer grid than the scanning.

We use spatial resolution and contrast ratio (CR) metrics to measure the performance of the beamformers in the study. The spatial resolution is measured by the full-width-at-halfmaximum (FWHM) of single-point targets. Generally, the smaller the value of FWHM or speckle size, the better spatial resolution the beamformer achieves. The CR between a lesion and the background is given by

$$
\mathrm{CR}=\frac{\left|I_{\text {out }}-I_{\text {in }}\right|}{\sqrt{I_{\text {out }}^{2}+I_{\text {in }}^{2}}},
$$

where $I_{\text {in }}$ and $I_{\text {out }}$ are the log-compressed mean intensities inside and outside the lesion, respectively. It has a value that varies from 0 to 1 with improving image contrast. To minimize the influence of the attenuation and diffraction of the ultrasound, we choose the measuring kernel as one circle inside the lesion and one ring around the lesion.

\section{B. Simulation Study}

We begin by evaluating the beamformer performance on the simulation dataset. This is generated with three pointscatterers, located at depths of $17.5 \mathrm{~mm}, 20 \mathrm{~mm}$, and $22.5 \mathrm{~mm}$ and a lateral position of $4 \mathrm{~mm}$, embedded in the random background. There is also a $5 \mathrm{~mm}$ diameter circular hypoechoic lesion centered at a depth of $20 \mathrm{~mm}$ and at $-4 \mathrm{~mm}$ in lateral position.

Beamformed images with dynamic focusing, F-DMAS, Unified PB and F-UDMAS are showed in Figs. 3, respectively. In the figure, both F-DMAS and Unified PB are showed to improve the lateral resolution compared to the dynamic focusing (Figs. 3(b) and (c) versus (a)). In addition, the unified PB generates a uniform image with clear speckle patterns. Meanwhile the F-DMAS is shown to have a significant positive impact on the image contrast. By combining the two beamformers together, we form the F-UDMAS that generates the image with the best lateral resolution (Fig. 3(d)). It also maintains the high contrast resolution that is observed with the F-DMAS. To quantify the improvement, we measure the FWHMs of the lateral responses at the three scatterers and calculate the $\mathrm{CR}$ on the idealised lesion. The results are summarized in Table I.

\section{Phantom Study}

We demonstrate the beamformers on experimental data acquired with the Verasonics sytem by scanning a multipurpose multi-tissue phantom (model 040GSE, CIRS, Norfolk, VA, USA). The phantom has a sound speed of $1540 \pm 10 \mathrm{~m} / \mathrm{s}$, and its attenuation coefficient slope is $-0.5 \mathrm{~dB} /(\mathrm{cm} \cdot \mathrm{MHz})$. The scanned region includes three fine wire targets located at different depths and two hyperechoic circular lesions $8 \mathrm{~mm}$ in diameter, centered at a depth of approximately $30 \mathrm{~mm}$. The contrast of the two hyperechoic lesions are about $15 \mathrm{~dB}$ and $6 \mathrm{~dB}$, respectively.

The images generated by the beamformers are displayed in Figs. 4. Similar to the simulation study, both F-DMAS and unified PB outperform dynamic focusing in terms of lateral resolution. The F-DMAS generally improves the image contrast, but its brightness around the focal depth is much higher than that at other depths. The proposed F-UDMAS combines the strengths of both unified PB and F-DMAS, further boosting the lateral resolution. It also enhances the coherence of signals collected in the regions away from the focal depth, thus the generated image has more uniform contrast over the entire imaging region compared to F-DMAS (Figs. 4(d) versus (b)). We measure the FWHMs of lateral profiles of the responses to the three point-scatterers and the CRs calculated on the left lesion. The results are summarized in Table I. The improvements in the F-UDMAS images show the robustness of the combined algorithm. 


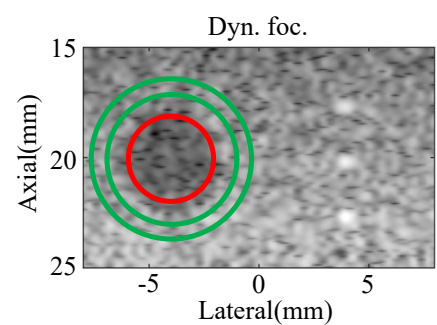

(a)

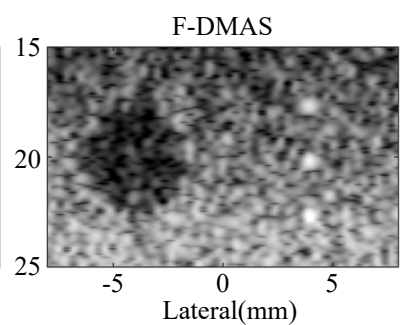

(b)

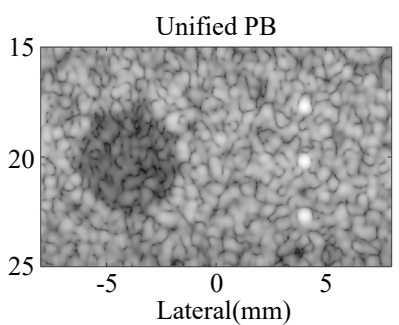

(c)

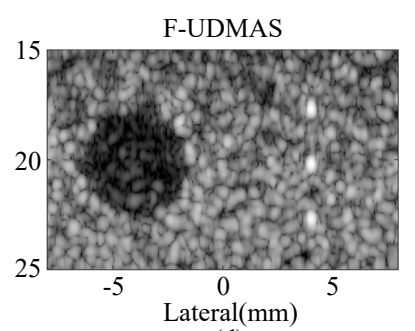

(d)

Fig. 3. Simulated images generated with different beamformers: (a) Dynamic focusing (Dyn. foc.), (b) F-DMAS, (c) Unified PB and (d) F-UDMAS. All pixel-based images are generated with data from 32 transmits. They are log-compressed and displayed with a dynamic range of $80 \mathrm{~dB}$.

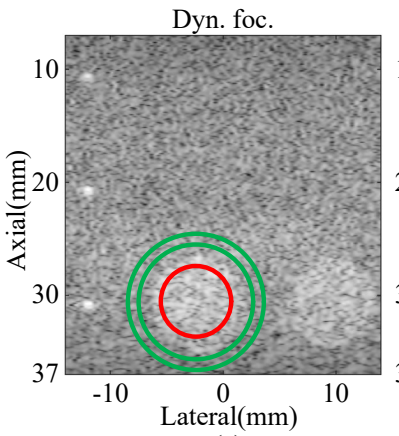

(a)

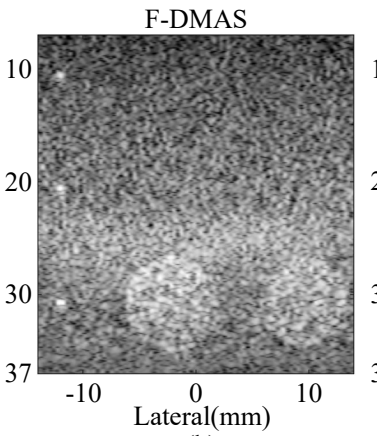

(b)

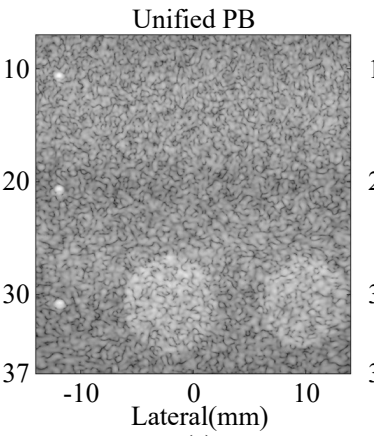

(c)

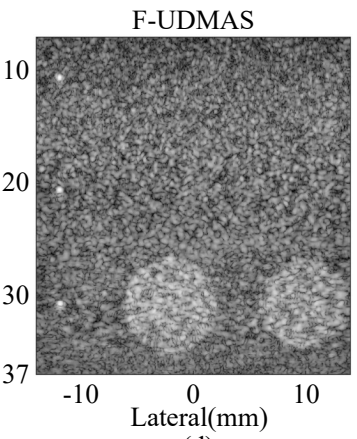

(d)

Fig. 4. Experimental images generated with different beamformers: (a) Dynamic focusing (Dyn. foc.), (b) F-DMAS, (c) Unified PB and (d) F-UDMAS. All pixel-based images are generated with data from 32 transmits. They are log-compressed and displayed with a dynamic range of $70 \mathrm{~dB}$.

TABLE I

PERFORMANCE METRICS ON SIMULATED AND EXPERIMENTAL IMAGES

\begin{tabular}{|c|c|c|c|c|c|c|c|c|}
\hline \multirow{2}{*}{ Beamformer } & \multicolumn{4}{|c|}{ Simulation } & \multicolumn{4}{c|}{ Phantom Study } \\
\cline { 2 - 4 } & \multicolumn{3}{|c|}{ FWHM (Lateral) at } & \multirow{2}{*}{ CR } & \multicolumn{3}{c|}{ FWHM (Lateral) at } & \multirow{2}{*}{ CR } \\
\cline { 2 - 6 } \cline { 6 - 8 } & $17.5 \mathrm{~mm}$ & $20 \mathrm{~mm}$ & $22.5 \mathrm{~mm}$ & & $11 \mathrm{~mm}$ & $21 \mathrm{~mm}$ & $31 \mathrm{~mm}$ & \\
\hline Dyn. foc. & 0.336 & 0.489 & 0.524 & 0.286 & 0.507 & 0.550 & 0.643 & 0.148 \\
\hline DMAS & 0.241 & 0.327 & 0.416 & 0.576 & 0.338 & 0.389 & 0.603 & 0.157 \\
\hline Unified PB & 0.336 & 0.350 & 0.375 & 0.293 & 0.393 & 0.444 & 0.490 & 0.151 \\
\hline F-UDMAS & $\mathbf{0 . 2 4 1}$ & $\mathbf{0 . 2 5 3}$ & $\mathbf{0 . 2 6 1}$ & $\mathbf{0 . 6 1 3}$ & $\mathbf{0 . 2 3 0}$ & $\mathbf{0 . 2 9 6}$ & $\mathbf{0 . 3 1 5}$ & $\mathbf{0 . 2 3 1}$ \\
\hline
\end{tabular}

\section{CONCLUSION}

We have developed a novel beamformer by combining the data generation in F-DMAS with the time-delay calculation from the unified PB beamformer. By doing so, we can enhance the spatial coherence information in the generated image, especially in the near- and far-field regions. We demonstrate the new beamformer F-UDMAS on both simulated and experimental data. The imaging results show the new F-UDMAS outperforms other existing strategies in terms of both lateral and contrast resolution. The generated image also has more uniform brightness over the entire imaging region. In the next step of this study we will consider the blurring artefacts caused by the transducer impulse response and the excitation pulse and extend the method to further improve the axial resolution.

\section{ACKNOWLEDGMENT}

This work was partially supported by the National Natural Science Foundation of China (NSFC61771130).

\section{REFERENCES}

[1] K. E. Thomenius, "Evolution of ultrasound beamformers," in Proceedings of the IEEE Ultrasonics Symposium, vol. 2, 1996, pp. 1615-1622.

[2] J. A. Jensen, S. I. Nikolov, K. L. Gammelmark, and M. H. Pedersen, "Synthetic aperture ultrasound imaging," Ultrasonics, vol. 44, pp. e5-e15, 2006.
[3] J. S. Hwang and T. K. Song, "A study of the display pixelbased focusing method in ultrasound imaging," Ultrasonic imaging, vol. 23, no. 1, pp. 1-18, 2001.

[4] N. Q. Nguyen and R. W. Prager, "High-resolution ultrasound imaging with unified pixel-based beamforming," IEEE transactions on medical imaging, vol. 35, no. 1, pp. 98-108, 2015.

[5] G. Matrone, A. S. Savoia, G. Caliano et al., "The delay multiply and sum beamforming algorithm in ultrasound b-mode medical imaging," IEEE transactions on medical imaging, vol. 34, no. 4, pp. 940-949, 2014.

[6] G. Matrone, A. S. Savoia, G. Caliano et al., "Depthof-field enhancement in filtered-delay multiply and sum beamformed images using synthetic aperture focusing," Ultrasonics, vol. 75, pp. 216-225, 2017.

[7] F. Prieur, O. M. H. Rindal, and A. Austeng, "Signal coherence and image amplitude with the filtered delay multiply and sum beamformer," IEEE transactions on ultrasonics, ferroelectrics, and frequency control, vol. 65, no. 7, pp. 1133-1140, 2018.

[8] J. A. Jensen and N. B. Svendsen, "Calculation of pressure fields from arbitrarily shaped, apodized, and excited ultrasound transducers," IEEE transactions on ultrasonics, ferroelectrics, and frequency control, vol. 39, no. 2, pp. 262-267, 1992. 